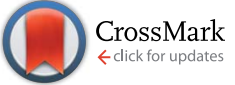

Cite this: RSC Adv., 2017, 7, 7310

Received 16th December 2016 Accepted 13th January 2017

DOI: $10.1039 / c 6 r a 28280 f$

www.rsc.org/advances

\section{Valleytronics of III-V solid solutions for thermoelectric application}

\author{
Payam Norouzzadeh, ${ }^{a}$ Ali Shakouri ${ }^{\mathrm{b}}$ and Daryoosh Vashaee ${ }^{\star a}$ \\ It is shown that the degeneracy of the bandstructure has different impacts on thermoelectric properties of \\ III-V materials. A comprehensive list of III-V solid solution alloys that can make multi-valley bandstructures \\ is presented and the transport parameters affecting their thermoelectric power factor are discussed. It is \\ shown that in some cases, such as $\mathrm{Ga}_{x} \mathrm{In}_{1_{-} \mathrm{P}} \mathrm{P}$ ternary alloy, the power factor is enhanced significantly \\ near the valley convergence point, but in some other cases, such as $\mathrm{Al}_{x} \mathrm{Ga}_{1-x} \mathrm{As}$, the enhancement is \\ marginal and away from the valley convergence point. Therefore, in addition to the bandstructure \\ engineering, detailed calculation of the transport parameters is also required to determine the optimum \\ alloy composition that can improve the thermoelectric properties.
}

The engineering and control over the valley degree of freedom is known as valleytronics, which has found many applications in semiconductors. ${ }^{1-4}$ The valleytronics is also a powerful approach for engineering high performance thermoelectric materials. Recent advances in thermoelectric materials has been mainly resulted from the reduction of the lattice thermal conductivity mainly by nanostructuring, ${ }^{5}$ or optimization of materials with complex unit cells. ${ }^{6,7}$ As the lattice thermal conductivity reduces and approaches the electronic thermal conductivity, the figure-of-merit $(z T)^{8}$ enhancement becomes less significant and other directions that increase the thermoelectric power factor are necessary for further improvement of the $z T$. The power factor enhancement can be achieved by various methods such as improvement of the density of states (DOS), ${ }^{9-12}$ creation of sharp changes in the DOS near the Fermi energy, ${ }^{13}$ increase of the number of carrier pockets near the Fermi energy, ${ }^{14}$ engineering composite structures, ${ }^{15,16}$ hot electron filtering via superlattices ${ }^{17,18}$, impurity-induced band structure distortion through the introduction of resonant energy levels close to the band edges, ${ }^{19,20}$ modulation doping approach which makes use of the enhancement in carrier mobility, ${ }^{21}$ tuning the doping and composition ${ }^{22}$ and engineering the carrier pockets near the Fermi energy. ${ }^{23}$ Most of these efforts are focused on enhancing of the power factor through modification of the DOS. In particular, it is known that a high valley degeneracy leads to large Seebeck coefficient, which may lead to a high power factor and $z T .^{24-27}$ Therefore, it is highly desired to find practical means to manipulate the valleys in the band structure. Among different techniques, solid solution of miscible materials is known as a method for tuning

${ }^{a}$ Electrical and Computer Engineering Department, North Carolina State University, Raleigh, NC 27606, USA. E-mail: dvashae@ncsu.edu; Tel: +1 (919) 530-9599 ${ }^{b}$ Birck Nanotechnology Center, Purdue University, West Lafayette, IN 47907, USA the bandstructure characteristics in order to move the band extrema close to each other. ${ }^{28}$ For this, the respective bands must have multiple valleys located close to each other ( $<$ a few $\left.k_{\mathrm{B}} T\right)$. However, it is also known that the carrier mobility can be reduced due to the intervalley scattering at the valleys convergence point. A key question is raised here: does the maximum power factor occur where the valleys converge? In this work, we aimed to address the mentioned question and elucidate on the multi-valley effect on thermoelectric transport properties in III$\mathrm{V}$ alloys. We present a comprehensive survey of III-V materials that simultaneously satisfy both the solid solution condition and the ability to make multiple valleys in the conduction band. The detailed transport calculations are presented for two selected alloys and a list of other candid materials is proposed for further studies.

Solid solutions are alloys in which one or more types of components may be partially substituted by the other components while maintaining similar structure. They are homogenous and the lattice structure remains unaffected at different composition ratios. To determine the degree of solubility, one may apply Hume-Rothery rules, ${ }^{29}$ which indicate: (a) the difference in atom radii of the solute and the solvent should not exceed $15 \%$, (b) the crystal structures of the solute and the solvent must be the same, (c) the electronegativity of the solute and the solvent must match, and finally (d) the valency of the solute and the solvent should be the same for complete solubility.

The initial idea is to use valleytronics for improving the thermoelectric power factor. This can be achieved by engineering the band structures that favor multiple valley electronic conduction. Solid solution of two materials having band minima with dissimilar wavenumbers can provide the roadmap for achieving this goal at or near the valleys convergence point. The number of carrier pockets involved in transport increases 
near the transition point; hence, for a given carrier concentration, the Fermi energy reduces, which results in the enhancement of Seebeck coefficient. If this enhancement happens without loss of the electrical conductivity, the thermoelectric power factor increases. To reach our goal, the alloy must satisfy two conditions: (a) the composition should be adjusted so that the different valleys converge. In the case of solid solution, the volume fraction is simply the "knob" for tuning the energy of the valleys and fulfilling the condition; (b) the conduction band minimum of material $1\left(\mathrm{CBM}_{1}\right)$ should be at a different wavenumber than that of material $2\left(\mathrm{CBM}_{2}\right)$ so that the band crossing happens. The later condition is required only if the bowing factor of the resulted bandstructure is negligible and the bands do not cross. For example, Fig. 1 shows the schematics of the bandstructure with conduction band minima at $L$, $\Gamma$, and $X$ (panel a) and the band-gap energies $\left(E_{\Gamma}, E_{L}\right.$, and $\left.E_{X}\right)$ as a function of the $x$-composition parameter for $\mathrm{Ga}_{x} \mathrm{In}_{1-x} \mathrm{P}$ (panel b).$^{30}$ The arrows in panel (a) indicate the intervalley electron scattering among different valleys.

In this ternary alloy, the conduction band minimum makes transition from $\Gamma$ to $L$ point at $x \approx 0.67$ and again from $L$ to $X$ point at $x \approx 0.77$ as the Ga concentration increases from zero to one. Therefore, the band degeneracy at $x \approx 0.67$ is five-fold and at $x \approx 0.77$ is 7 -fold. The latter is even larger than the degeneracy of the conduction band in some of the good thermoelectric materials like $\mathrm{Bi}_{2} \mathrm{Te}_{3}$ and SiGe (both 6-fold degenerate). It is
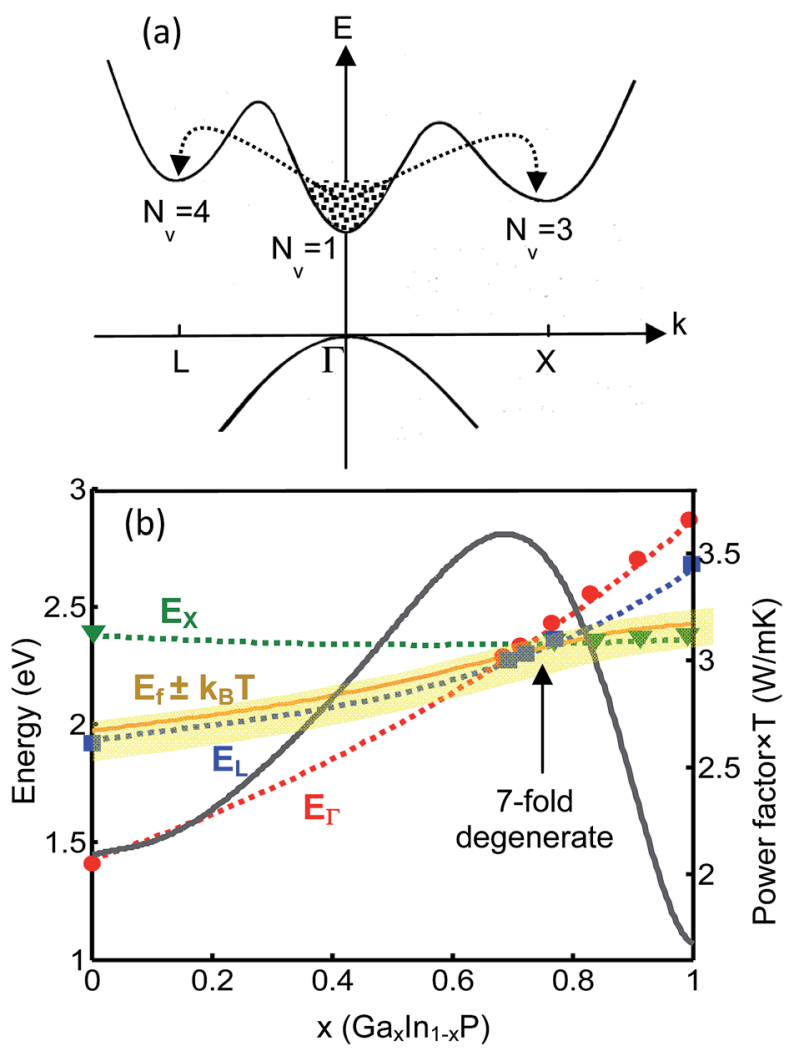

Fig. 1 (a) Illustration of a multivalley band structure with valleys $L, \Gamma$, and $X$. (b) Conduction band minima vs. $x$ for $G a_{x} \mid n_{1-x} P$. The power factor is calculated for doping concentration of $1.5 \times 10^{20} \mathrm{~cm}^{-3}$ at $600{ }^{\circ} \mathrm{C}$. Symbols represent the experimental data. ${ }^{28}$ worth noting that $E_{\Gamma}$ value at $x \approx 0.77$ is very close to $E_{L}$ and $E_{X}$. Therefore, at temperatures that $k_{\mathrm{B}} T$ is comparable to or larger than the energy difference, the $T$ valley also contributes in the carrier transport, adding to the $X$ and $L$ contributions, which would further enhance the Seebeck coefficient.

We calculated the main thermoelectric properties of $n$ type $\mathrm{Ga}_{x} \mathrm{In}_{1-x} \mathrm{P}$ by solving the Boltzmann Transport Equation. For the model calculations, we followed the methodologies discussed in our previous works. ${ }^{31-34}$ The calculation details as well as the model validations by the experimental data are presented in the supplementary material available in the online version of this article. The calculation was performed for fixed doping concentration of $N=1.5 \times 10^{20} \mathrm{~cm}^{-3}$ at $T=600{ }^{\circ} \mathrm{C}$. This value of $N$ maximizes the $z T$ at the optimum $x$. As shown in Fig. 2, the relatively large value of the degeneracy of the band in this material system has resulted in significant enhancement of the Seebeck coefficient. The peak power factor occurs at $x \approx 0.7$, which is near both the $T-L$ and $L-X$ band convergence points. At this range of composition, $I, L$ and $X$ valleys are near each other (within a $k_{\mathrm{B}} T$ ). Therefore, there are effectively 8 carrier pockets at the band edge. When the $I, L$ and $X$ are within several $k_{\mathrm{B}} T$ from each other, the carriers can populate all the valleys and contribute in electronic transport. The electrical and thermal transport properties are calculated and shown in Fig. 2. In order to show the effect of the intervalley scattering, we have plotted the values corresponding to two case of with or without the inclusion of the intervalley scattering. Alloying of InP with Ga reduces the electron mobility; ${ }^{35}$ however, the effect in Seebeck coefficient is stronger and results in significant enhancement of the power factor, as shown in Fig. $2 \mathrm{a}$ and b. Our calculations also show that the multi-band transport has significantly reduced the thermal conductivity, which is mainly due to the reduction of the electronic part of the thermal conduction (the thermal conductivity components are not shown in the plot). Therefore, the combination of the two mechanisms predicts a high $z T$ close to 1.1 near $x \approx 0.74$.

In contrast to $\mathrm{Ga}_{x} \mathrm{In}_{1-x} \mathrm{P}$ material system, our calculations for $\mathrm{n}$ type $\mathrm{Al}_{x} \mathrm{Ga}_{1-x} \mathrm{As}$ shows that the optimum bandstructure, i.e. where the $z T$ is maximized, deviates from the valley convergence point. The calculated thermoelectric properties for $\mathrm{Al}_{x} \mathrm{Ga}_{1-x} \mathrm{As}$ versus $x$ at $600{ }^{\circ} \mathrm{C}$ is shown in Fig. 3. The doping concentration was fixed at $N=1.05 \times 10^{19} \mathrm{~cm}^{-3}$, which maximizes the $z T$ at optimum $x$.

In this case, the band extrema $L$ and $X$ converge at $x \approx 0.42$. It is generally expected that the $z T$ should increase near this point. However, model calculations predict that $z T$ reaches a peak at $x \approx 15 \%$ and it reduces significantly as $x$ approaches 0.42 . This different trend indicates that the bands convergence is not necessarily the optimum place at which $z T$ is enhanced. The main reason for the $z T$ reduction near the valley convergence point is due to the significant drop of the electrical conductivity near this point (Fig. 3a). In general, the valley convergence affects the transport properties in two ways. First, it enhances the density of states; hence, improves the Seebeck coefficient. Second, it increases the intervalley charge carrier scattering, which reduces both the electrical conductivity and the Seebeck coefficient. ${ }^{36}$ The dominant scattering near the 

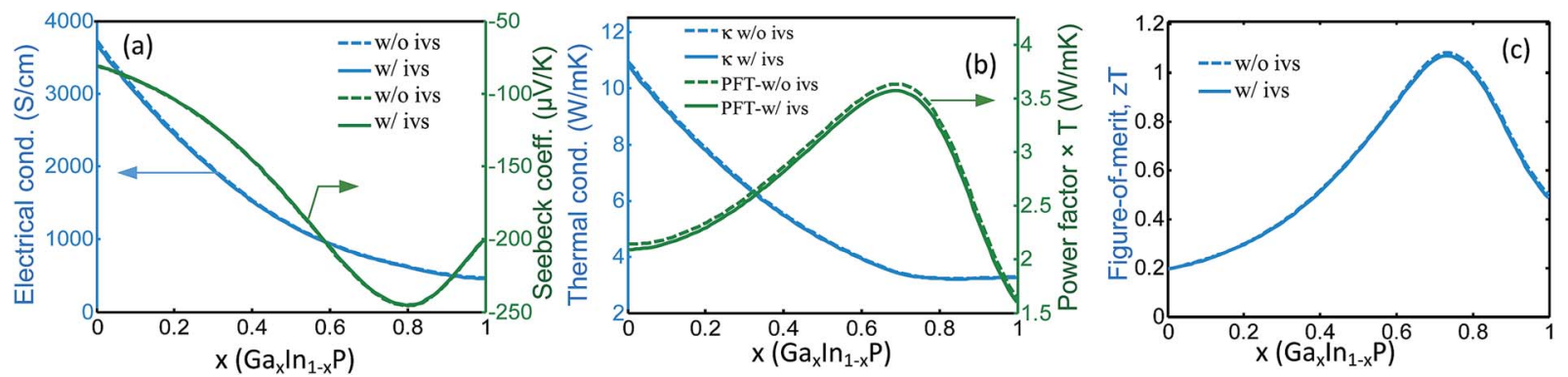

Fig. 2 Theoretical prediction of (a) electrical conductivity and Seebeck coefficient, (b) thermal conductivity and power factor, and (c) $z T$ versus $\mathrm{Ga}$ fraction $x$ at $600^{\circ} \mathrm{C}$ for $\mathrm{Ga}_{x} \mid \mathrm{n}_{1-x} \mathrm{P}$. Solid and dashed lines show the values corresponding to two cases as with intervalley scattering (w/ ivs) and without intervalley scattering (w/o ivs). All plots are calculated for doping concentration of $N=1.5 \times 10^{20} \mathrm{~cm}^{-3}$ at $600{ }^{\circ} \mathrm{C}$.
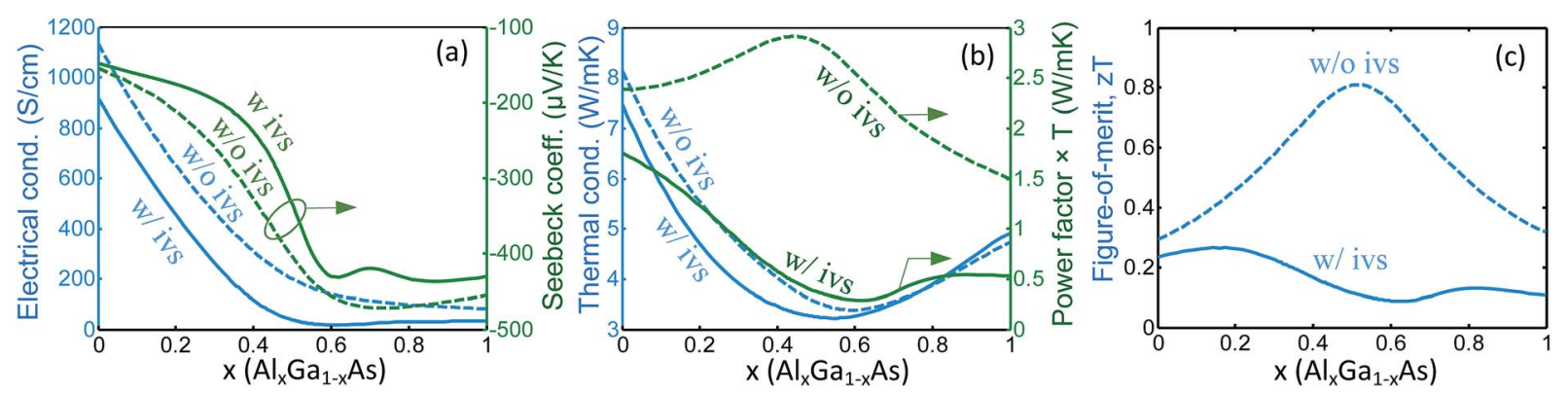

Fig. 3 Theoretical prediction of (a) electrical conductivity and Seebeck coefficient, (b) thermal conductivity and power factor, and (c) zT versus $\mathrm{Ga}$ fraction $x$ at $600{ }^{\circ} \mathrm{C}$ for $\mathrm{Al}_{x} \mathrm{Ga}_{1-x} \mathrm{As}$. Solid and dashed lines show the values corresponding to two cases as with intervalley scattering (w/ ivs) and without intervalley scattering (w/o ivs). All plots are calculated for doping concentration of $N=1.05 \times 10^{19} \mathrm{~cm}^{-3}$ at $600{ }^{\circ} \mathrm{C}$.

band convergence point in both materials is intervalley scattering; however, the intervalley scattering in $\mathrm{Al}_{x} \mathrm{Ga}_{1-x} \mathrm{As}$ is more significant. Therefore, in $\mathrm{Al}_{x} \mathrm{Ga}_{1-x} \mathrm{As}$, although the Seebeck coefficient is improved, the reduction of carrier mobility is dominant and the power factor reduces at and near the valley convergence point. For the case of $\mathrm{Ga}_{x} \mathrm{In}_{1-x} \mathrm{P}$, the former effect (Seebeck coefficient) is dominant and the power factor enhances at the valley convergence point. As illustrated in Fig. 3 b, the power factor is greatly affected by intervalley scattering. At presence of the intervalley scattering, the power factor shows a minimum at $x \approx 0.6$, while upon exclusion of the intervalley scattering it has a peak at $x \approx 0.5$. In this case, in contrast to $\mathrm{Ga}_{x} \mathrm{In}_{1-x} \mathrm{P}$, the multi-valley transport has some small effect on the thermal conductivity (Fig. 3b). The ambipolar thermal conductivity is small as in $\mathrm{Ga}_{x} \mathrm{In}_{1-x} \mathrm{P}$ and is not shown in the figures. The overall effect of the valley convergence on $z T$, as depicted in Fig. 3c, is similar to the behavior of the power factor. $z T$ has a peak value of $z T \approx 0.8$ at $x \approx 0.55$ if the intervalley scattering is removed, and it shows a significant drop upon inclusion of the intervalley scattering and the peak value is $z T \approx 0.27$ at $x \approx 0.16$.

A comprehensive list of III-V solid-solution candidates for thermoelectric application is presented in Table 1. The band parameters were collected from ref. 37 and the valley convergence was calculated using Vegard's law by the inclusion of the bowing parameters whenever the data was available. The table shows the candid binary solid solution $\mathrm{A}_{x} \mathrm{~B}_{1-x}$, the lattice constant mismatch $\Delta a$, the bandgap $E_{\mathrm{g}}$ and the corresponding band-edge for material A and B, the composition $x_{1}$ where the bands meet and its corresponding degeneracy $N_{\mathrm{v} 1}$ and the valleys, the composition $x_{2}$ which has the highest number of carrier pockets near the band-edge and the corresponding degeneracy $N_{\mathrm{v} 2}$, and the overlap temperature $T_{\mathrm{o}}$. As an example, for the case of GaP-GaSb, i.e. $(\mathrm{GaP})_{x}$ $(\mathrm{GaSb})_{1-x}$, the lattice mismatch is \%11.8. The bandgap for $\mathrm{GaP}$ and $\mathrm{GaSb}$ are $2.35 \mathrm{eV}$ at $X$ point and $0.81 \mathrm{eV}$ at $\Gamma$ point, respectively. $X$ and $L$ valleys meet at $x \sim 0.42$ resulting in 7 fold degeneracy. At $x \sim 0.38$ and at $T \sim 140 \mathrm{~K}, \Gamma$ point is less than $2 k_{\mathrm{B}} T$ above the $X$ and $L$ points. Therefore, at $T>150 \mathrm{~K}$, the $\Gamma$ point becomes also populated and there will be 8 carrier pockets contributing in transport. The materials in the nonshaded rows are suggested as good candidates due to their high degeneracy of the band at the valley convergence point. For the first nine rows, at temperatures above $T_{\mathrm{o}}$, the $T, X$ and $L$ CBMs will all contribute in transport due to the broadening of the Fermi-Dirac distribution. This contribution in turn increases the number of carrier pockets near the Fermi energy. Interestingly, for GaP-GaSb, GaP-InP, InAs-AlAs, GaSbAlP, and GaAs-AlAs the overlapping temperature is below the room temperature.

The valley convergence can occur between any two valleys of $\Gamma, X$, and $L$. In such cases, $x_{1}$ composition corresponds to the valley convergence that results in highest degeneracy. The overlap temperature $T_{\mathrm{o}}$ refers to the temperature above which the energy separation of the three valleys of $I, X$, and $L$ becomes less than $2 k_{\mathrm{B}} T$. 
Table 1 List of investigated III-V solid solution alloys ${ }^{a}$

\begin{tabular}{llllll}
$\mathbf{A}_{\mathbf{x}} \mathbf{B}_{\mathbf{1}-\mathbf{x}}$ & $\mathbf{\Delta a}$ & $\mathbf{E g}(\mathbf{e V})$ & $\mathbf{x}_{\mathbf{1}}, \mathbf{N}_{\mathbf{v} 1}$ & $\mathbf{x}_{\mathbf{2}}, \mathbf{N}_{\mathbf{v} 2}$ & $\mathbf{T}_{\mathbf{0}}(\mathbf{K})$ \\
\hline GaP-GaSb & $\% 11.8(2.35, \mathrm{X})-(0.81, \Gamma)$ & $0.42,7(\mathrm{XL})$ & $0.38,8$ & 140 \\
GaP-InP & $\% 7.1$ & $(2.35, \mathrm{X})-(1.42, \Gamma)$ & $0.72,7(\mathrm{XL})$ & $0.70,8$ & 125 \\
InAs-AIAs & $\% 6.8$ & $(0.417, \Gamma)-(2.24, \mathrm{X})$ & $0.37,4(\mathrm{X} \Gamma)$ & $0.37,8$ & 161 \\
GaSb-AIP & $\% 11$ & $(0.812, \Gamma)-(2.45, \mathrm{X})$ & $0.77,4(\mathrm{X} \Gamma)$ & $0.77,8$ & 202 \\
GaAs-AIAs & $\% 0.18$ & $(1.52, \Gamma)-(2.24, \mathrm{X})$ & $0.58,4(\mathrm{X} \Gamma)$ & $0.58,8$ & 48 \\
GaAs-AISb & $\% 8.5$ & $(1.52, \Gamma)-(1.70, \mathrm{X})$ & $0.60,4(\mathrm{X} \Gamma)$ & $0.60,8$ & 897 \\
GaP-GaAs & $\% 3.5$ & $(2.35, \mathrm{X})-(1.52, \Gamma)$ & $0.45,4(\mathrm{X} \Gamma)$ & $0.45,8$ & 554 \\
GaSb-AISb & $\% 0.6$ & $(0.812, \Gamma)-(1.70, \mathrm{X})$ & $0.53,4(\mathrm{X} \Gamma)$ & $0.67,8$ & 487 \\
GaP-InAs & $\% 11$ & $(2.35, \mathrm{X})-(0.42, \Gamma)$ & $0.65,4(\mathrm{X} \Gamma)$ & $0.65,8$ & 805 \\
InSb-AISb & $\% 5.5$ & $(0.24, \Gamma)-(1.70, \mathrm{X})$ & $0.54,4(\mathrm{X} \Gamma)$ & - & 2636 \\
InAs-AISb & $\% 1.2$ & $(0.42, \Gamma)-(1.70, \mathrm{X})$ & $0.40,4(\mathrm{X} \Gamma)$ & - & 1485 \\
InP-AIP & $\% 7$ & $(1.42, \Gamma)-(2.52, \mathrm{X})$ & $0.62,4(\mathrm{X} \Gamma)$ & - & 2336 \\
GaP-AISb & $\% 3.8$ & $(2.35, \mathrm{X})-(1.70, \mathrm{X})$ & - & - & - \\
GaP-AIP & $\% 0.1$ & $(2.35, \mathrm{X})-(2.52, \mathrm{X})$ & - & - & - \\
GaP-AIAs & $\% 3.7$ & $(2.35, \mathrm{X})-(2.24, \mathrm{X})$ & - & - & - \\
GaAs-InP & $\% 3.7$ & $(1.52, \Gamma)-(1.42, \Gamma)$ & - & - & - \\
AlAs-AISb & $\% 8$ & $(2.24, \mathrm{X})-(1.70, \mathrm{X})$ & - & - & - \\
AIP-AISb & $\% 11.6(2.52, \mathrm{X})-(1.70, \mathrm{X})$ & - & - & - \\
AIP-AIAs & $\% 3.6$ & $(2.52, \mathrm{X})-(2.24, \mathrm{X})$ & - & - & -
\end{tabular}

${ }^{a} \Delta a$ : the lattice mismatch. $E_{\mathrm{g}}$ : band gap. The corresponding valley is also shown. $x_{1}$ and $N_{\mathrm{v} 1}$ : the composition where two valleys meet and the corresponding valley degeneracy. $x_{2}$ and $N_{\mathrm{v} 2}$ : the composition with highest number of contributing electron pockets in transport at temperatures above $T_{\mathrm{o}}$, and the corresponding number of carrier pockets. $T_{\mathrm{o}}$ : the temperature above which $L, X$, and $\Gamma$ valleys can be populated at optimum Fermi energy. The band parameter data is taken from Vurgaftman $e t$ al. $^{37}$

Therefore, all the three bands, $\Gamma, L$, and $X$ contribute effectively in the transport. It is also worth mentioning that the mentioned materials have zinc blende structure, and except GaP-GaSb and GaP-InP, the valley convergence occurs at $X / L$ cross point. GaP-GaSb and GaP-InP are especially interesting as they have the highest degeneracy at $x_{1}$. The convergence of $X$ and $L$ valleys at CBM in these two solid solutions is due to their large bandstructure bowing parameters. ${ }^{37}$ For the three alloys in the lightly shaded area of the table, there is no $X L$ valley convergence and the overlap temperature is above the melting point. For the remaining seven alloys in the darkly shaded area, there is neither $T L$ nor $X L$ valley convergence.

As discussed earlier, the high degeneracy of the band is only one of the criteria for a good thermoelectric material. The nondominancy of the intervalley scattering must be also satisfied in order to observe the improvement of the thermoelectric power factor by multi-valley transport conduction. If the intervalley scattering becomes significant near the valley convergence point, the optimum alloy composition can deviate away from this point or the $z T$ may be reduced. For example, as shown in Fig. 3, the optimum $x$ parameter for n-type $\mathrm{Al}_{x} \mathrm{Ga}_{1-x}$ As deviates from where the valleys meet each other $(x \approx 0.42)$ and happens at $x \approx 0.16$. Therefore, the compositions listed in.

Table 1 does not necessarily maximize the power factor.

In summary, it was shown that valleytronics has different impacts on thermoelectric properties of III-V alloys. A comprehensive list of materials that can make a continuous solid solution with high valley degeneracy were identified. The continuous solid solution of these alloys provide means for tuning the valley characteristics of the band structure. The solid solution of two materials having band minima with dissimilar wavenumbers allows convergence of the valleys and enhancing the valley degeneracy $\left(N_{\mathrm{v}}\right)$, hence, the electronic density of states. The enhancement of the density of states generally improves the Seebeck coefficient providing a roadmap for engineering high performance thermoelectric materials. However, it was also discussed that the enhancement of the density of state may be accompanied by large intervalley scattering that can deviate the optimum alloy composition away from the valley convergence point or reduce the $z T$. Therefore, as the next step, the transport calculations are required for the valleytronics of these candid alloys in the search for good thermoelectric materials.

\section{Acknowledgements}

This study is partially based upon work supported by Air Force Office of Scientific Research (AFOSR) under contract number FA9550-12-1-0225 and the National Science Foundation (NSF) under grant numbers EEC-1160483, ECCS-1351533 and CMMI1363485.

\section{References}

1 A. Popescu and L. M. Woods, Adv. Funct. Mater., 2012, 22, 18.

2 K. Behnia, Nat. Nanotechnol., 2012, 7, 488-489.

3 C. E. Nebel, Nat. Mater., 2013, 12, 690-691.

4 D. Culcer, A. L. Saraiva, B. Koiller, X. Hu and S. D. Sarma, Phys. Rev. Lett., 2012, 108, 126804.

5 A. Chernatynskiy, D. R. Clarke and S. R. Phillpot, Handbook of Nanoscience, Engineering, and Technology, CRC Press, 3rd edn, 2012.

6 G. J. Snyder and E. S. Toberer, Nat. Mater., 2008, 7, 105-114.

7 C. Toher, J. J. Plata, O. Levy, M. de Jong, M. Asta, M. Buongiorno Nardelli and S. Curtarolo, Phys. Rev. B: Condens. Matter Mater. Phys., 2014, 90, 174107.

8 H. J. Goldsmid, Introduction to Thermoelectricity, Springer, Heidelberg, Germany, 2010.

9 T. C. Harman, P. J. Taylor, M. P. Walsh and B. E. LaForge, Science, 2004, 303, 818.

10 L. D. Hicks and M. S. Dresslhaus, Phys. Rev. B: Condens. Matter Mater. Phys., 1993, 47, 16631.

11 Y. Ma, Q. Hao, B. Poudel, Y. C. Lan, B. Yu, D. Z. Wang, G. Chen and Z. F. Ren, Nano Lett., 2008, 8, 2580.

12 K. F. Garrity, Phys. Rev. B, 2016, 94, 045122.

13 J.-H. Lee, J. Wu and J. C. Grossman, Phys. Rev. Lett., 2010, 104, 016602.

14 P. Norouzzadeh, C. W. Myles and D. Vashaee, Sci. Rep., 2014, 7028, DOI: $10.1038 /$ srep07028.

15 D. J. Bergman and L. G. Feldman, J. Appl. Phys., 1999, 85, 8205.

16 J. P. Heremans, V. Jovovic, E. S. Toberer, A. Saramat, K. Kurosaki, A. Charoenphakdee, S. Yamanaka and G. J. Snyder, Science, 2008, 25, 554. 
17 D. Vashaee and A. Shakouri, Phys. Rev. Lett., 2004, 92, 106103.

18 J. M. O Zide, D. Vashaee, G. Zeng, J. E. Bowers, A. Shakouri and A. C. Gossard, Phys. Rev. B: Condens. Matter Mater. Phys., 2006, 74, 205335.

19 K. Ahn, M. Han, J. He, J. Androulakis, S. Ballikaya, C. Uher, V. P. Dravid and M. G. Kanatzidis, J. Am. Chem. Soc., 2010, $132(14), 5227$.

20 B. Yu, Q. Zhang, H. Wang, X. Wang, H. Wang, D. Wang, H. Wang, G. J. Snyder, G. Chen and Z. F. Ren, J. Appl. Phys., 2010, 108, 016104.

21 M. Zebarjadi, G. Joshi, G. Zhu, B. Yu, A. Minnich, Y. Lan, X. Wang, M. Dresselhaus, Z. Ren and G. Chen, Nano Lett., 2011, 11(6), 2225.

22 Y. Pei, X. Shi, A. LaLonde, H. Wang, L. Chen and G. J. Snyder, Nature, 2011, 473, 66.

23 P. Norouzzadeh, C. W. Myles and D. Vashaee, J. Phys.: Condens. Matter, 2013, 25(47), 475502.

24 G. D. Mahan, Solid State Physics, Academic, 1998.

25 H. J. Goldsmid, Thermoelectric Refrigeration, Plenum, 1964.

26 G. A. Slack, Handbook of Thermoelectrics, CRC, 1995, ch. 34.

27 F. J. DiSalvo, Science, 1999, 285, 703.
28 Y. Tang, Z. M. Gibbs, L. A. Agapito, G. Li, H.-S. Kim, M. Nardelli, S. Curtarolo and G. J. Snyder, Nat. Mater., 2015, 4430.

29 M. Uichiro, Hume-Rothery Rules for Structurally Complex Alloy Phases, Taylor \& Francis, 2010.

30 D. Auvergne, P. Merle and H. Mathieu, Solid State Commun., 1997, 21, 5 .

31 A. J. Minnich, H. Lee, X. W. Wang, G. Joshi, M. S. Dresselhaus, Z. F. Ren, G. Chen and D. Vashaee, Phys. Rev. B: Condens. Matter Mater. Phys., 2009, 80, 155327.

32 P. Norouzzadeh and D. Vashaee, J. Electron. Mater., 2015, 44, 2.

33 N. Satyala and D. Vashaee, J. Electron. Mater., 2012, 499(1), 68-1791.

34 N. Satyala, P. Norouzzadeh, and D. Vashaee, Nano Bulk Thermoelectrics: Concepts, Techniques, and Modeling, Nanoscale Thermoelectrics, ed. X. Wang and Z. M. Wang, Springer, 28 October 2013, ch. 4, ISBN: 978-3-319-02011-2 (Print) 978-3-319-02012-9 (Online).

35 H. M. Macksey, N. Holonyak Jr, R. D. Dupuis, J. C. Campbell and G. W. Zack, J. Appl. Phys., 1973, 44(3), 1333-1341.

36 P. Norouzzadeh and D. Vashaee, Sci. Rep., 2016, 6, 22724, DOI: $10.1038 /$ srep22724.

37 I. Vurgaftman and J. R. Meyer, J. Appl. Phys., 2001, 89, 5815. 